Published in final edited form as:

Tech Coloproctol. 2019 April ; 23(4): 325-332. doi:10.1007/s10151-019-01951-w.

\title{
Progression of Anal Intraepithelial Neoplasia in HIV-Positive Individuals: Predisposing Factors
}

\author{
Tonna McCutcheon, DNP*, Alexander T Hawkins, MD, MPH ${ }^{\star}, \#$, Roberta L Muldoon, MD*, M \\ Benjamin Hopkins, MD*, Timothy M Geiger, MD*, and Molly M Ford, MD* \\ *Vanderbilt University Medical Center, Department of General Surgery, Colon and Rectal Surgery, \\ Nashville, TN, USA
}

\#Authors McCutcheon and Hawkins have contributed equally and are co-first authors.

\begin{abstract}
Background: The aim of the present study was to evaluate patient factors that affect the progression of anal dysplasia in human immunodeficiency virus (HIV)-positive individuals.

Methods: A retrospective cohort study of HIV-positive adults with anal dysplasia were identified from 2012 to 2017. Patient files were reviewed and data was collected. A bivariate analysis of continuous and categorical variables was performed.
\end{abstract}

Results: One hundred sixty-one patients met inclusion criteria. Ninety-seven percent were male. Mean age was 41 years. Thirty-five percent were African American and 47\% were Caucasian. Nine percent had progression of disease. Presence of visible lesions (therefore no anal pap test) was associated with progression. A lower initial CD4 count $(\mathrm{p}=0.01)$ and initial surgical pathology of anal condylomata $(\mathrm{p}=0.01)$ were also associated with progression. High-risk serotype was associated with no change or regression $(\mathrm{p}=0.01)$.

Conclusions: Most notably, visible lesions (no anal pap) and CD4 count when lower were associated with progression. Initial surgical pathology of anal condylomata was associated with progression while high-risk serotypes correlated with regression or stability. Identification of risk factors has important implications concerning postoperative surveillance and counseling of HIVpositive patients with anal condylomata/anal dysplasia.

\section{Keywords}

anal dysplasia; anal intraepithelial neoplasia; AIN progression

Address for correspondence: Alexander T Hawkins, MD, MPH, Section of Colon \& Rectal Surgery, Vanderbilt University, $116121^{\text {st }}$ Ave South, Room D5248 MCN, Nashville, TN 37232, USA, Office: 615.343.4612, Fax: 615.343.4615, alex.hawkins@ vumc.org. Disclaimer: There are no conflicts of interest or off-label use

Presented as a poster at the American Society of Colon and Rectal Surgeons, Annual Meeting, May 19-23, 2018, Nashville, TN, USA Conflict of interest: the authors declare that they have no conflict of interest 


\section{Introduction}

Although rare, the incidence of anal carcinoma has gradually increased over the past three decades, with a reported occurrence of 1 per 100,000 individuals within the general population[1]. However, human immunodeficiency virus (HIV) -positive individuals have a higher rate of development of anal carcinoma, with a reported incidence of 60-160 per 100,000 in HIV-positive men who have sex with men (MSM) [1]. This select population also has been shown to have higher rates of progression or recurrence of anal dysplasia [2].

Human papilloma virus (HPV) is an identifiable source in approximately $93 \%$ of anal carcinomas, with the most common associated high-risk oncogenic HPV strains being HPV 16 and 18 [3,4]. These strains generate changes in the squamous epithelium, leading to anal intraepithelial neoplasia (AIN), which is a precursor to anal carcinoma [5].

The natural history of anal dysplasia is not fully understood. It is suggested that anal dysplasia develops in a progressive manner from onset of AIN 1 through AIN 3 and eventually to anal carcinoma [5]. Co-existing factors may influence the rate of progression along the neoplastic spectrum.

The purpose of this study was to evaluate patient factors that may affect the rate of progression of levels of anal dysplasia within the HIV-positive population. We hypothesize that there are important and potentially modifiable factors associated with progression of anal dysplasia.

\section{Materials and methods}

\section{Study design}

We performed a retrospective cohort study of all consecutive HIV-positive adults undergoing an exam under anesthesia with pathologic findings at a tertiary care hospital from January 1, 2012 to November 1, 2017. Exclusion criteria were as follows: patients without follow-up pathology or clinical exam, patients with initial diagnosis of squamous cell carcinoma, patients with a CD4 count $<200$ cells/ $\mu \mathrm{L}$ and patients who were HIV-negative. (Figure 1) Of note, our practice does not in general operate on patients with a CD4 count lower than 200 cells $/ \mu$ as previous data has indicated that patients diagnosed with autoimmune deficiency syndrome (AIDS) have significant issues with wound healing when undergoing anorectal surgery [14].

On presentation, a complete history was taken and a complete physical examination was carried out, including digital rectal examination and standard anoscopy. Treatment of patients seen in our clinic for AIN was based upon an existing protocol:those with grossly visible lesions on clinic anoscopy would be scheduled for surgical excision and fulguration in the operating room, as well as those without visible lesions in clinic who had an anal pap showing HSIL because our level of suspicion was high. Suspicious visible lesions were defined as smooth papular, keratotic, or white plaque-like growths in the anal canal or perianal skin. High resolution anoscopy (HRA) was not utilized, since this procedure is not an institutional protocol. To date, there has been no definitive data that has suggested HRA 
is superior to expectant management with close observation in the prevention of anal cancer. The American Society of Colorectal Surgeons (ASCRS) indicates HRA is a consideration for screening in high risk patients if performed by trained providers, but the recommendation is a weak recommendation based on moderate-quality evidence as outlined in the ASCRS clinical practice guidelines [6]. The specimens were sent to the pathology department at Vanderbilt University Medical Center. Each tissue sample was reviewed by two pathologists, with a low threshold for further evaluation within the group. There are five gastrointestinal pathologists at our institution. If the tissue was equivocal for high-grade lesions, a p16 stain was obtained. Pathology results were categorized as normal, anal condylomata (AC), AIN 1 (mild dysplasia), AIN 2 (moderate dysplasia), and AIN 3 (severe dysplasia) [7]. The authors did not distinguish between patients with AIN 2 and a positive p 16 stain and patients with AIN 2 and negative p16 stain. All AIN 2 patients were considered high-risk and were surveillanced as such. Patients with a histologic diagnosis of anal condylomata or AIN 1 were scheduled to undergo surveillance with anoscopy every six months and patients with AIN 2 or AIN 3 were scheduled to undergo surveillance with anoscopy every 3 months. All patients with a histologic diagnosis of AIN 1, 2, or 3 were offered use of imiquimod cream postoperatively for 16 weeks (Figure 2). Patients with recurrence of disease underwent excision and fulguration of lesions. For the purposes of this study, the interval between exams was defined as the time between the first surgery and the second surgery or the next follow-up exam.

Patients were divided into two groups, those that progressed and those that did not. Progression was defined as a higher grade of AIN or progression to squamous cell carcinoma on follow up surgical pathology. Patients with a clinical exam without any identifiable lesions were included in the non-progression group. Factors associated with progression were identified. The Vanderbilt University Institutional Review Board (Protocol 170799) reviewed and approved this study waiving informed consent. The study was also conformed to the preferred reporting of case series in surgery (PROCESS)checklist [8].

\section{Data collection}

Patient files were retrospectively reviewed and pertinent demographic, pathologic and surgical information was collected. Study data were collected and managed using REDCap electronic data capture tools hosted at Vanderbilt University Medical Center [9]. REDCap (Research Electronic Data Capture) is a secure, web-based application designed to support data capture for research studies. Covariates collected include: age, gender, ethnicity, presence of high-risk HPV serotypes, history of Gardasil (one, two, or three doses), history of sexually transmitted infections, HIV status, use of imiquimod postoperatively (defined as patient was given a prescription for 16 weeks of imiquimod cream), initial CD4 count (indicator of the patient's immunosuppression at the time of initial surgery), initial viral load, tobacco use, patient reported history of anal or genital condylomata, patient reported history of anal receptive intercourse, patient reported number of lifetime sexual partners, patient reported use of condoms, initial anal cytology, and initial surgical pathology. An additional covariate was the presence of a visible lesion on outpatient anal examination. If lesions were large enough to be detected on anal examination, then patients often did not undergo an anal pap test as it would not have changed the plan for surgical excision. 


\section{Statistical analysis}

Continuous and categorical variables are expressed as mean with standard deviation and proportions throughout the study, with the exception of continuous variables with grossly skewed distributions that are reported as the median with the interquartile range. Bivariate analysis of continuous and categorical variables was performed with either the Student's Ttest or the Wilcoxon rank-sum test depending on distribution and Fishers exact test respectively. For analysis of the clinical characteristics in relation to initial surgical pathology, proportional odd logistic regression modeling was used given the ordinal nature of initial surgical pathology. SAS statistical software (version 9.3; SAS Institute's Inc., Cary NC, USA) was used for all analyses. All tests were two sided with an alpha level of 0.05 .

\section{Results}

Over the study period, $161 \mathrm{HIV}$-positive patients met the inclusion criteria and were analyzed. Of the entire group, 156 (97\%) were male, 76 (47\%) Caucasian, and the median age was 41 years (range 30-50 years). (Table 1) The mean initial CD4 count was 604 cells per microliter and $15 \%$ of patients had a detectable initial viral load. Fifty percent of patients tested positive for high-risk HPV. The median interval between the initial surgery and the second surgery or next follow up visit was 331 days.

Progression was observed in 14 (9\%) of patients, and 2 patients (1\%) progressed to cancer (Figure 3). A number of factors were associated with progression (Table 1). Patients who did not have initial anal pap cytology, but underwent examination under anesthesia (EUA) due to the presence of visible lesions alone were found to have progression of disease (Progression: 57\%; No Progression: 24\%; $\mathrm{p}=0.02$ ). Initial CD4 counts were significantly lower in the group that experienced disease progression (Progression: 387; No Progression: 619; $\mathrm{p}=0.02$ ) and those with a diagnosis of anal condylomata on initial surgical pathology (Progression: $62 \%$; No Progression: $16 \%$; $=0.001$ ) were more likely to progress. Patients with high-risk HPV serotypes (Progression: 23\%; No Progression: 54\%; $\mathrm{p}=0.03$ ) were less likely to progress. There was no difference observed in median interval between exams among the two groups (Progression: 404 days; No Progression: 303 days; $\mathrm{p}=0.38$ ).

In an analysis of clinical characteristics in relation to initial surgical pathology, only two factors were associated with increasing grades of dysplasia. (Table 2) : both increasing age (OR 1.02; 95\% CI 1.00-1.05) and tobacco use (OR 2.13; 95\% CI 1.19-3.81)

\section{Discussion}

In this retrospective cohort study, we evaluated HIV-positive patients with anal dysplasia looking for demographic or clinical factors associated with progression of anal dysplasia. An important finding was the high incidence of progression in patients with no initial anal pap cytology, but with visible lesions on exam at time of referral. Another notable finding was that patients with progression had a significantly lower initial CD4 count. Also, patients with anal condylomata on surgical pathology were found to have a higher risk of disease progression. Interestingly, we also noted that high-risk HPV serotypes were associated with a lower progression and that time to follow up did not seem to make a difference. 
Progression of AIN is likely influenced by co-existing factors. Past research has indicated risks include anal receptive intercourse, the presence of oncogenic HPV infection, tobacco use, immunocompromised state, and abnormal anal cytology [5, 10]. Other reported risk factors for the progression of AIN have been mentioned in recent studies and include CD4 counts below 200 cells per microliter, a previous history of anal carcinoma, and MSM over than 40 years of age $[5,11,12]$.

Patients in our cohort with no initial anal pap performed, but with visible lesions on exam had a higher rate of progression. No previous studies address visible lesions without associated cytology specifically. These patients also were found to have a high rate of AIN 3 ( $41 \%$ of the group of AIN 3) on initial exam, and it has been suggested that the rate of progression of high grade perianal AIN to carcinoma is as high as $18 \%$ [12]. These results would suggest that surgical intervention, if lesions are visualized, is a more appropriate treatment than initial management with topical medications. This suggestion may be supported by an open-label randomized trial conducted by Richel et al comparing electrocautery to 5 fluorouracil and imiquimod. The author's findings suggested electrocautery was a better treatment option for AIN than 5 fluorouracil or imiquimod [13].

In general, a low CD4 count has a known association with risk of acquiring opportunistic infections. However, we specifically demonstrated a difference in CD4 counts in patients who progress, as they had a significantly lower mean initial CD4 count. Progression with a very low CD4 count has been suggested in the past by Tinmouth et al, who found that a diagnosis of acquired immunodeficiency syndrome (AIDS) was associated with progression to carcinoma [12]. But our study looked at all patients who progressed and found that their mean CD4 count was 387 cells per microliter, versus the non-progressors or regressors with a CD4 count mean of 619. This should encourage providers to be more aggressive with surveillance regimens in patients with lower CD4 counts. Of note, our practice does not in general operate on patients with a CD4 count lower than 200 cells per microliter as previous data has indicated that patients diagnosed with AIDS have significant issues with wound healing when undergoing anorectal surgery [14].

Our outcomes also suggested that anal condylomata had an elevated risk of disease progression compared to higher grade AIN. Fazendin et al. found similar results and reported anal condylomata and low-grade dysplasia exhibited a high risk of progression to moderate-grade dysplasia with surveillance by anoscopy every 6 to 12 months [15]. Patients within our study with a surgical pathology of anal condylomata, as well as AIN 1, were under planned surveillance with anoscopy every 6 months as compared to patients with AIN 2 or AIN 3 who were under planned surveillance with anoscopy every 3 months. However, despite our recommendations for follow-up, there was not a significant difference in followup periods between what we had initially deemed the low risk group (condylomata, AIN1) and the high risk group (AIN 2 and 3).

The overall rate of disease progression from AIN to anal cancer remains unclear. However, one reported estimation suggested a $1.3 \%$ to $3.2 \%$ rate of progression within a 5-year period $[10,16]$. The rate of progression from AIN 1 to AIN 2 or AIN 3 to carcinoma is less well understood, but since many AIN 1 lesions are believed to be caused by low-risk HPV strains, 
the risk of progression to high-grade dysplasia and carcinoma has historically been thought to be lower. However, a counterintuitive finding in our study was a significant decrease in progression with identified high-risk HPV strains, meaning those without identified highrisk HPV showed progression. Although the literature is not definitive regarding this topic, Fazendin et al also found that HPV genotypes did not affect the rate of progression of dysplasia [15]. This contrasts with Pokomandy et al who suggested the risk of progression from a low-grade dysplasia to a high-grade dysplasia was greater in HIV-positive MSM older than 40 years of age with positive oncogenic HPV [11]. Similarly, Burgos et al reported HIV-positive MSM with high-risk HPV serotypes were found to have a two-fold increase in the risk of progression to high-grade dysplasia [5]. Our findings of decreased progression may be related to the overall low number of patients with disease progression or to more aggressive initial treatment for patients with identified high-risk HPV, going straight to surgical excision.

Although the findings were not statistically significant, it is important to mention that patients who had at least one dose of Gardasil were found to have no progression of disease (Progression: 0\%; No Progression: 14\%; $\mathrm{p}=0.22$ ). The low number of patients receiving Gardasil may be a factor in the insignificant findings. However, this information supports further study of the Gardasil vaccine in this setting.

The strengths of our study include the examination of a large non-HRA population, evaluation of patients with no initial anal pap cytology with visible lesions present on exam, analysis of CD4 counts in relationship to progression of AIN disease, and assessment of the effect of Gardasil on progression of AIN disease. To date, few studies have been done without the use of HRA and within this non-HRA study, no progression to anal carcinoma was found. Therefore, our findings may in fact suggest the acceptability of visual examination of the perianal and anal regions, which may prove to be less costly to patients as well as health care institutions.

HRA has been championed by some as the optimal modality for anal dysplasia screening [17]. Our institutional protocol does not include HRA in the treatment algorithm for AIN disease. Rather, the inspection of the anal canal with use of anoscopy on a regimented basis is utilized to visualize lesions and aid in the prevention of disease progression. To date, minimal data exists regarding the superiority of HRA or expectant management in prevention of progression of AIN disease. Crawshaw et al. compared HRA to expectant management in surveillance of AIN and prevention of anal carcinoma [18]. Results indicated that out of 424 patients (220 managed with HRA and 204 managed with expectant management), 3 patients progressed to anal carcinoma. Of the 3 patients, 2 were noncompliant with follow-up and HIV management and 1 was allergic to imiquimod, which was used postoperatively [18]. The researchers concluded the development of anal cancer is rare despite the method of treatment if patients are compliant with protocols [18]. Therefore, the importance of regimented surveillance and compliance with HIV treatment may be more of a factor as compared to the method of surveillance.

Limitations of this study include the low rate of progression that precluded building a multivariable model to assess risk factors. Because of this, we are unable to assess 
interaction between covariates in the progression of AIN lesions. Also, this study examined the progression of overall AIN disease without emphasis on specific AIN lesions. This study was limited in that the data was obtained from a single institution and the majority of the population was male. Therefore, results may not be generalizable to other populations affected by AIN disease. Furthermore, this study looked at baseline data and did not track changes in CD4 counts and tobacco use over the course of the study. Finally, this select population of study was challenging in terms of continued surveillance. Many patients missed appointments or were lost to care, which in turn limited our ability to adequately examine time as a factor in the progression of AIN disease. Further studies that focus on the location of lesions in relationship to progression of disease may impart additional knowledge that could potentially drive future treatment for AIN disease.

In summary, factors that were shown to affect disease progression include visible lesions, low CD4 counts, anal condylomata on surgical pathology, absence of high risk HPV serotypes and gender. As a result of these findings, we will modify our present practice by offering surgical intervention as a first line treatment for visible lesions as well as provide additional counseling for patients with surgical pathology demonstrating anal condylomata about the risk of progression of disease and the importance of continued surveillance, shortening this interval for patients with anal condylomata. And finally, while our data did not find significance with use of Gardasil and/or imiquimod postoperatively, we will continue to gather data and expand our initial database in the hopes of answering these questions. Now with prospective collection of the data we hope to provide greater longitudinal information with the eventual hope of generating a multivariate model to create a predictive model for disease progression.

\section{Conclusions}

Most notably, visible lesions with no associated anal pap cytology correlated with progression and patients that did progress had a significantly lower CD4 count. Initial surgical pathology of anal condylomata was associated with progression while high-risk serotypes correlated with regression or stability. At this time, there are no recommended surveillance guidelines or universal treatment protocols for this select population. Therefore, advocation for more aggressive surveillance intervals and treatment of visible lesions, anal condylomata and AIN disease is imperative.

\section{Supplementary Material}

Refer to Web version on PubMed Central for supplementary material.

\section{Acknowledgements}

This project utilized the REDCap database, which is maintained with grant support (UL1 TR000445 from NCATS/ $\mathrm{NIH})$.

Support: Internal funding 


\section{References}

1. Salit LE, Lytwyn A, Raboud J et al. (2010). The role of cytology (pap tests) and human papillomavirus testing in anal cancer screening. AID 24:1307-1313.

2. Goldstone SE, Johnstone AA, Moshier EL. (2014). Long-term outcome of ablation of anal highgrade squamous intraepithelial lesions: recurrence and incidence of cancer. Disease of the Colon and Rectum 57(3): 316-323.

3. Machalek DA, Poynten M, Jin F et al. (2012), Anal human papillomavirus infection and associated neoplastic lesions in men who have sex with men: A systematic review and meta-analysis. Lancet Oncology 13:487-500. [PubMed: 22445259]

4. The HPV and Anal Cancer Foundation. (2013). HPV. Available from: http:// www.analcancerfoundation.org/learn/hpv/

5. Burgos J, Curran A, Tallada N et al. (2015). Risk of progression to high-grade anal intraepithelial neoplasia in HIV-infected MSM. AIDS 29: 695-702. [PubMed: 25849833]

6. Stewart D, Gaertner W, Glasgow S, Herzig D, Feingold D, Steele S. (2018). The American Society of Colon and Rectal Surgeons Clinical Practice Guidelines for Anal Squamous Cell Cancers (Revised 2018). Diseases of the Colon and Rectum 61: 752-774.

7. Darragh TM, Colgan TJ, Cox JT et al. (2012). Members of LAST Project Work Groups. The Lower Anogenital Squamous Terminology Standardization Project for HPV-Associated Lesions: background and consensus recommendations from the College of American Pathologists and the American Society for Colposcopy and Cervical Pathology. J Low Genit Tract Dis 16: 205-242. [PubMed: 22820980]

8. Agha R, Fowler A, Rajmohan S, Barai I, Orgill D. (2016). Preferred reporting of case series in surgery; the PROCESS guidelines. International Journal of Surgery 36: 312-323. [PubMed: 27816703]

9. Harris PA, Taylor R, Thielke R, Payne J, Gonzalez N, Conde JG. (2009). Research electronic data capture (REDCap) - A metadata-driven methodology and workflow process for providing translational research informatics support. J Biomed Inform 42: 377-81. [PubMed: 18929686]

10. Machalek DA, Poynten M, Jin F et al. (2012),Anal human papillomavirus infection and associated neoplastic lesions in men who have sex with men: A systematic review and meta-analysis. Lancet Oncology 13: 487-500. [PubMed: 22445259]

11. Pokomandy A, Rouleau D, Ghattas G et al. (2011). HAART and progression to high-grade anal intraepithelial neoplasia in men who have sex with men and are infected with HIV. Clinical Infectious Diseases 52: 1174-1181. [PubMed: 21364075]

12. Tinmouth J, Peeva V, Henok A et al. (2016). Progression from perianal high-grade anal intraepithelial neoplasia to anal cancer in HIV-positive men who have sex with men. Disorders of the Colon and Rectum 59: 836-842.

13. Richel O, de Vries H, van Noesel C, Dijkgraff M, Prins J. (2013). Comparison of imiquimod, to pical fluorouracil, and electrocautery for the treatment of anal intraepithelial neoplasia in HIVpositive men who have sex with men: an open-label, randomized controlled trial. Lancet Oncol 4: 346-353.

14. Lord RVN.(1997). Anorectal surgery in patients infected with human immunodeficiency virus: factors associated with delayed wound healing. Annals of Surgery 226: 92-99. [PubMed: 9242343]

15. Fazendin EA, Crean AJ, Fazendin JM et al. (2017). Condyloma acuminatum, anal intraepithelial neoplasia, and anal cancer in the setting of HIV: do we really understand the risk? Diseases of the Colon and Rectum 60: 1078-1082. [PubMed: 28891852]

16. Cachay ER, Mathews WC. (2013). Human papillomavirus, anal cancer, and screening considerations among HIV-infected individuals. AIDS 15: 122-133.

17. Goldstone SE. (2010). High-resolution anoscopy is a crucial component of anal dysplasia screening. Diseases of the Colon and Rectum 53: 364-365. [PubMed: 20173491]

18. Crawshaw BP, Stein RAJ, Reynolds HL et al. (2015). High-resolution anoscopy or expectant management for anal intraepithelial neoplasia for the prevention of anal cancer: is there really a difference? Disease of the Colon and Rectum 58: 53-59. 


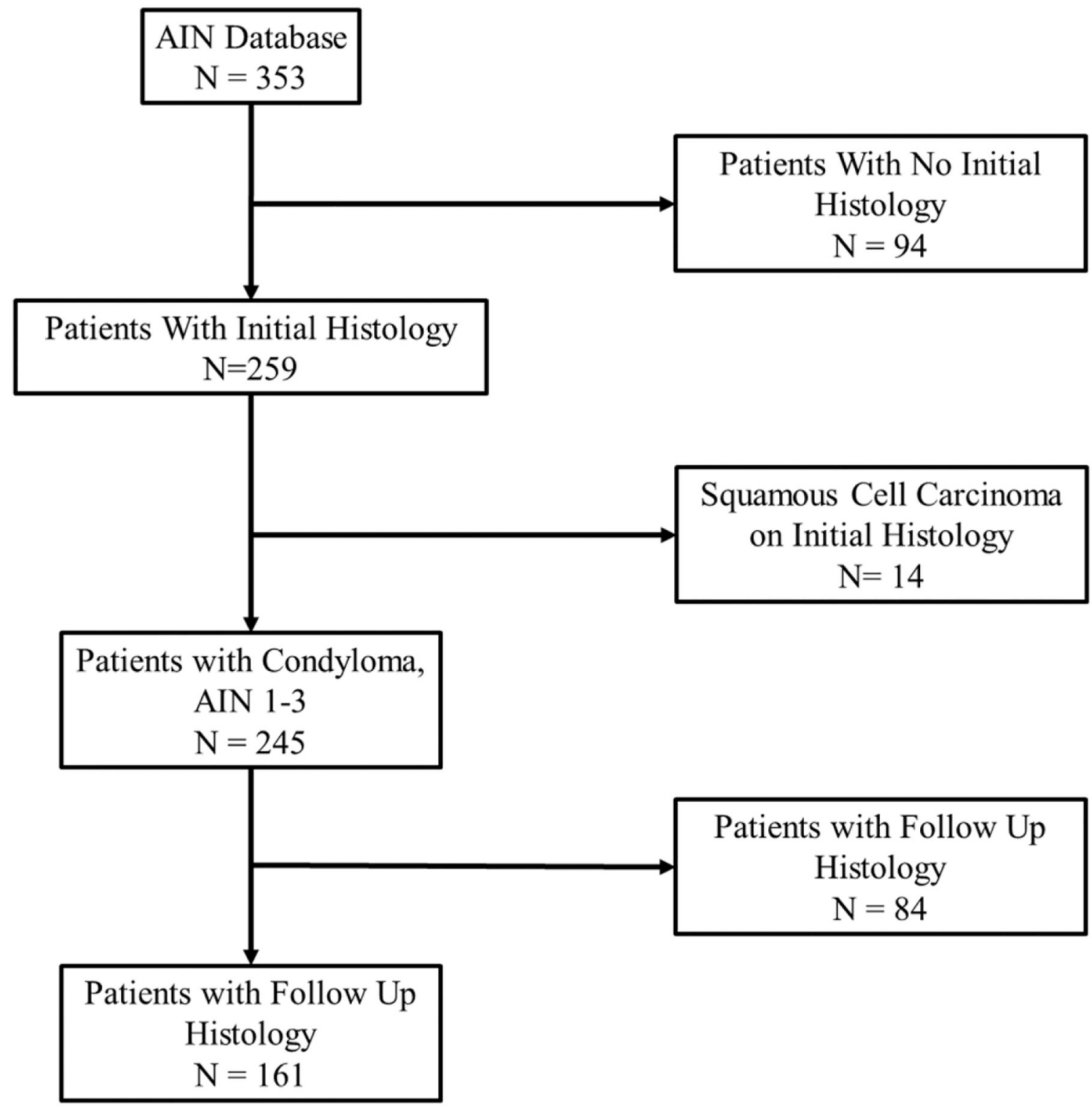

Figure 1-

Consolidated Standards of Reporting Trials (CONSORT) Diagram for Cohort Creation 


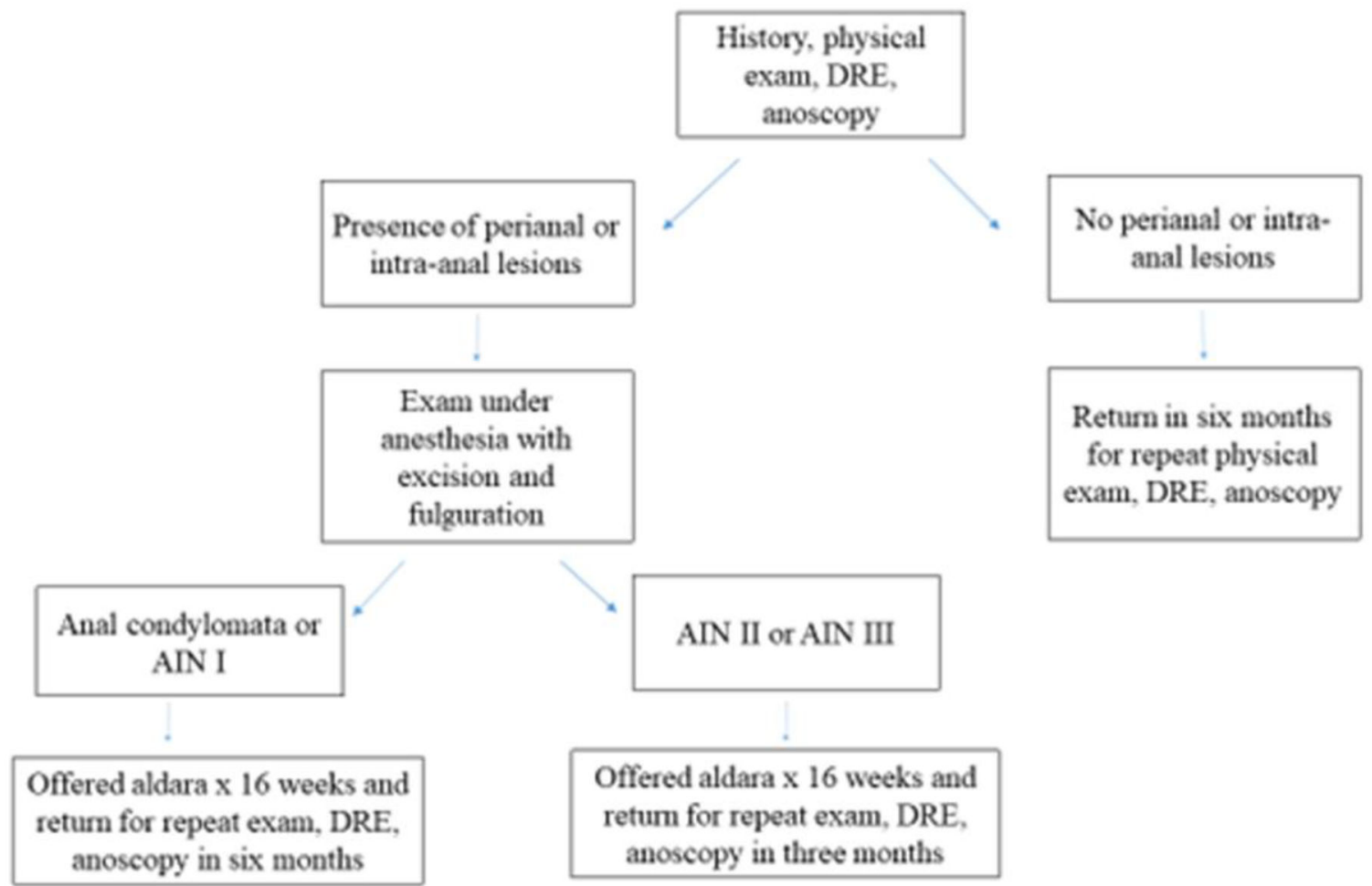

Figure 2-

Anal intraepithelial neoplasia (AIN) Surveillance Protocol 


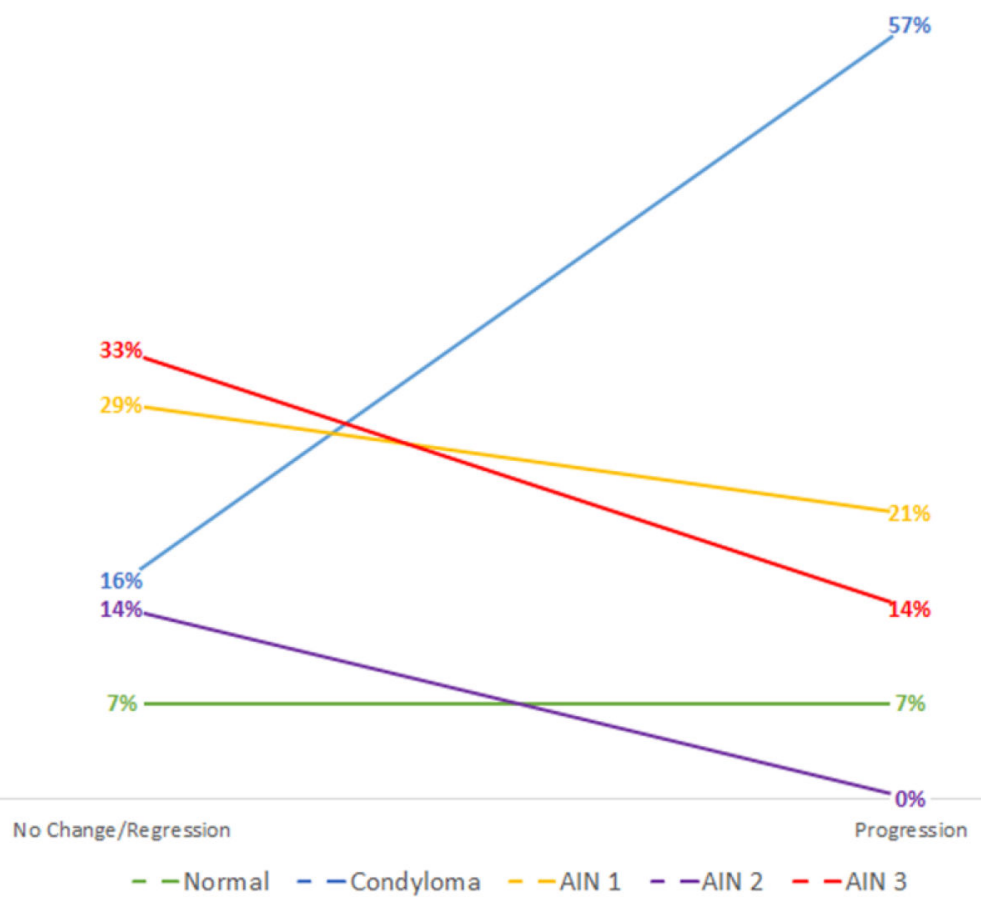

Figure 3-

Progression by Initial Surgical Pathology 
Table 1

Demographics and Progression of Disease

\begin{tabular}{|c|c|c|c|c|}
\hline Clinical Characteristics & All (n=161) & Progression $(n=14)$ & No Change/Regression $(n=147)$ & P-value \\
\hline Age, years, median & $41(30-50)$ & $44(35-54)$ & $41(30-50)$ & 0.17 \\
\hline Male Gender & $156(97 \%)$ & $12(86 \%)$ & $144(98 \%)$ & 0.01 \\
\hline Race & & & & 0.39 \\
\hline African American & $56(35 \%)$ & $7(50 \%)$ & $49(33 \%)$ & \\
\hline Caucasian & $76(47 \%)$ & $6(43 \%)$ & $70(47 \%)$ & \\
\hline Other Race & $29(18 \%)$ & $1(7 \%)$ & $28(19 \%)$ & \\
\hline High-risk HPV serotypes & $81(50 \%)$ & $3(23 \%)$ & $78(54 \%)$ & 0.03 \\
\hline History of Gardasil (1,2, or 3 doses) & $20(12 \%)$ & $0(0 \%)$ & $20(14 \%)$ & 0.22 \\
\hline History of STI & $74(46 \%)$ & $5(35 \%)$ & $69(47 \%)$ & 0.41 \\
\hline Use of imiquimod postoperatively & $54(33 \%)$ & $4(29 \%)$ & $50(34 \%)$ & 0.80 \\
\hline Initial CD4 count & $604(357-830)$ & $387(232-603)$ & $619(380-835)$ & 0.02 \\
\hline Detectable initial viral load & $25(15 \%)$ & $1(7 \%)$ & $24(16 \%)$ & 0.69 \\
\hline Tobacco use & $62(38 \%)$ & $6(43 \%)$ & $56(38 \%)$ & 0.90 \\
\hline History of anal or genital condylomata & $58(36 \%)$ & $7(50 \%)$ & $51(35 \%)$ & 0.40 \\
\hline Anal receptive intercourse & $62(38 \%)$ & $4(29 \%)$ & $58(40 \%)$ & 0.41 \\
\hline Lifetime sexual partners $>100$ & $7(4 \%)$ & $1(7 \%)$ & $6(4 \%)$ & 0.50 \\
\hline Use of condoms & $21(13 \%)$ & $1(7 \%)$ & $20(14 \%)$ & 0.69 \\
\hline Initial anal cytology & & & & 0.02 \\
\hline Initial anal cytology none & $44(27 \%)$ & $8(57 \%)$ & $36(24 \%)$ & \\
\hline Initial anal cytology ASCUS & $34(21 \%)$ & $1(7 \%)$ & $33(23 \%)$ & \\
\hline Initial anal cytology LSIL & $70(43 \%)$ & $5(36 \%)$ & $65(45 \%)$ & \\
\hline Initial anal cytology HSIL & $13(8 \%)$ & $0(0 \%)$ & $13(9 \%)$ & \\
\hline Initial surgical pathology & & & & 0.001 \\
\hline Initial surgical pathology normal & $11(7 \%)$ & $0(0 \%)$ & $11(7 \%)$ & \\
\hline Initial surgical pathology anal condylomata & $31(19 \%)$ & $8(62 \%)$ & $23(16 \%)$ & \\
\hline Initial surgical pathology AIN 1 & $47(29 \%)$ & $3(23 \%)$ & $43(29 \%)$ & \\
\hline Initial surgical pathology AIN 2 & $21(13 \%)$ & $0(0 \%)$ & $21(14 \%)$ & \\
\hline Initial surgical pathology AIN 3 & $51(32 \%)$ & $2(15 \%)$ & $49(33 \%)$ & \\
\hline
\end{tabular}

HPV=human papilloma virus; $\mathrm{STI}=$ sexually transmitted infection; ASCUS= atypical squamous cells of unknown significance; LSIL= low grade squamous intraepithelial lesion; HSIL= high grade squamous intraepithelial lesion; AIN= anal intraepithelial neoplasia 


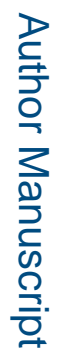

\begin{tabular}{|c|c|c|c|c|c|c|c|c|c|c|c|c|c|c|c|c|c|c|}
\hline 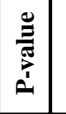 & 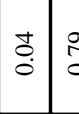 & : & & \begin{tabular}{l}
$\infty$ \\
\hdashline \\
\hdashline
\end{tabular} & 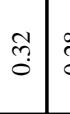 & 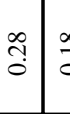 & 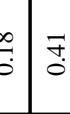 & $\stackrel{0}{\circ}$ & $\mid \begin{array}{l}0 \\
\dot{0}\end{array}$ & $\mid \begin{array}{l}\vec{n} \\
0\end{array}$ & $\mid$\begin{tabular}{c|c}
0 \\
0 \\
\cline { 1 - 1 } & 5 \\
\end{tabular} & $\mid \begin{array}{l}0 \\
0 \\
\end{array}$ & 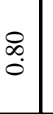 & & $\begin{array}{l}0 \\
0 \\
0 \\
0\end{array}$ & $\begin{array}{l}\infty \\
0 \\
0\end{array}$ & $\begin{array}{l}g \\
\text { of }\end{array}$ & $\stackrel{\text { ga }}{0}$ \\
\hline \begin{tabular}{c|c}
0 \\
8 \\
0 \\
0 \\
0 \\
0
\end{tabular} & 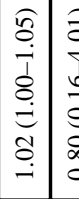 & $\begin{array}{l}\hat{\sigma} \\
\dot{b} \\
0 \\
\dot{b} \\
0 \\
0 \\
0\end{array}$ & 凯 & 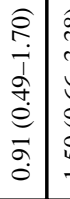 & 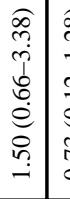 & 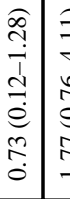 & 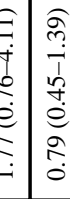 & 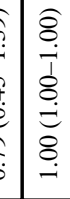 & 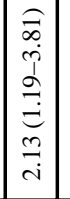 & 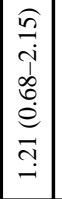 & 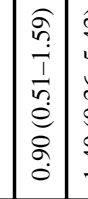 & 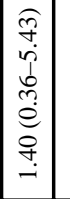 & 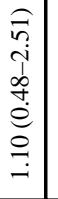 & 宦 & 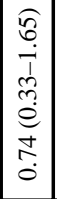 & 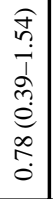 & 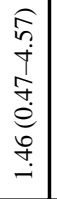 & 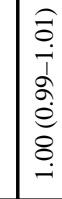 \\
\hline 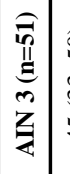 & 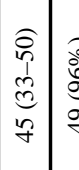 & & & 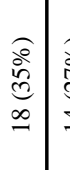 & 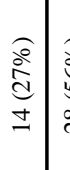 & 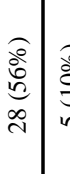 & 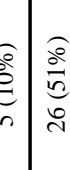 & 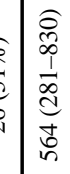 & 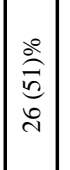 & 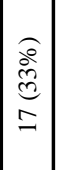 & 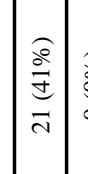 & 拿 & 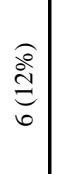 & 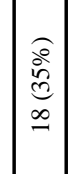 & 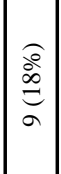 & ర్ర & 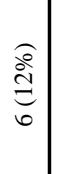 & $\begin{array}{l}6 \\
\infty \\
+ \\
0 \\
6\end{array}$ \\
\hline
\end{tabular}

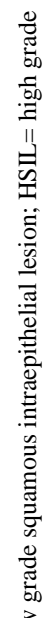



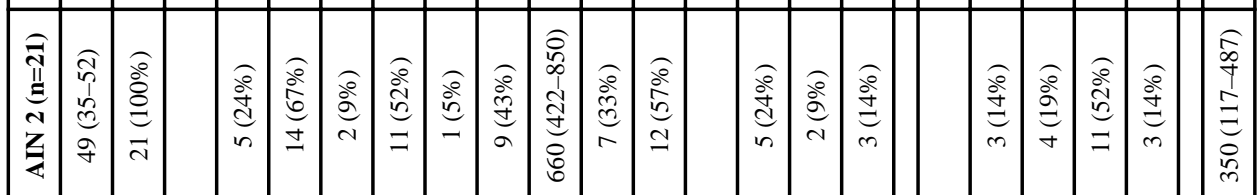

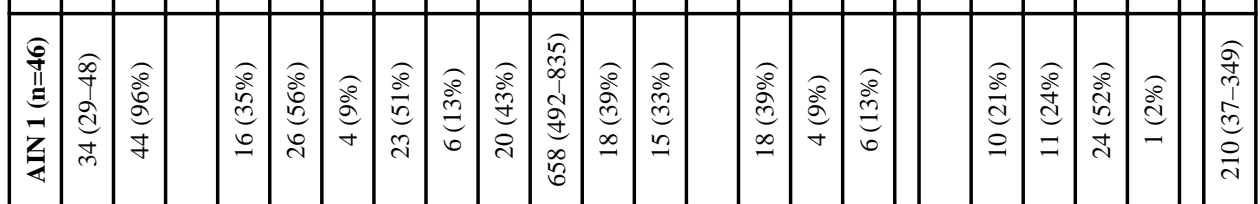

तิ

政

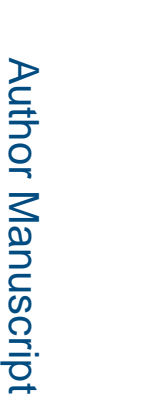

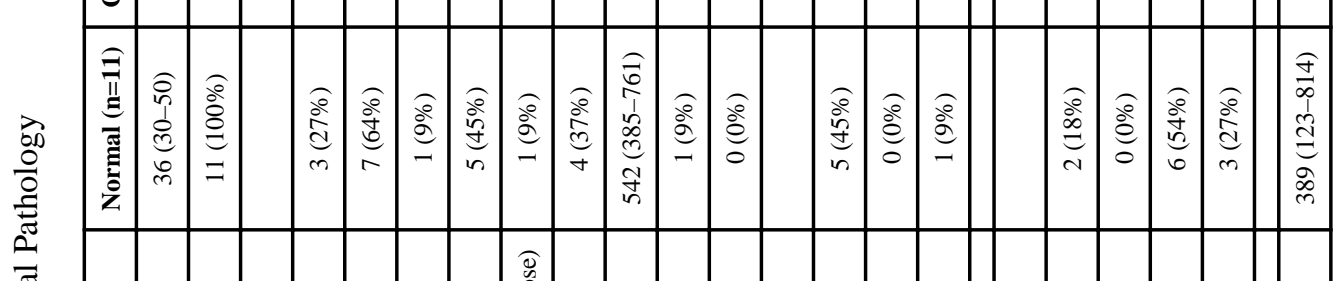

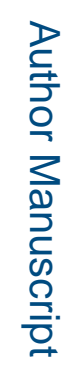

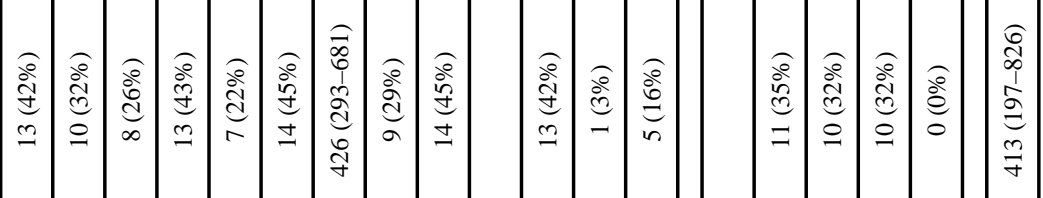
离

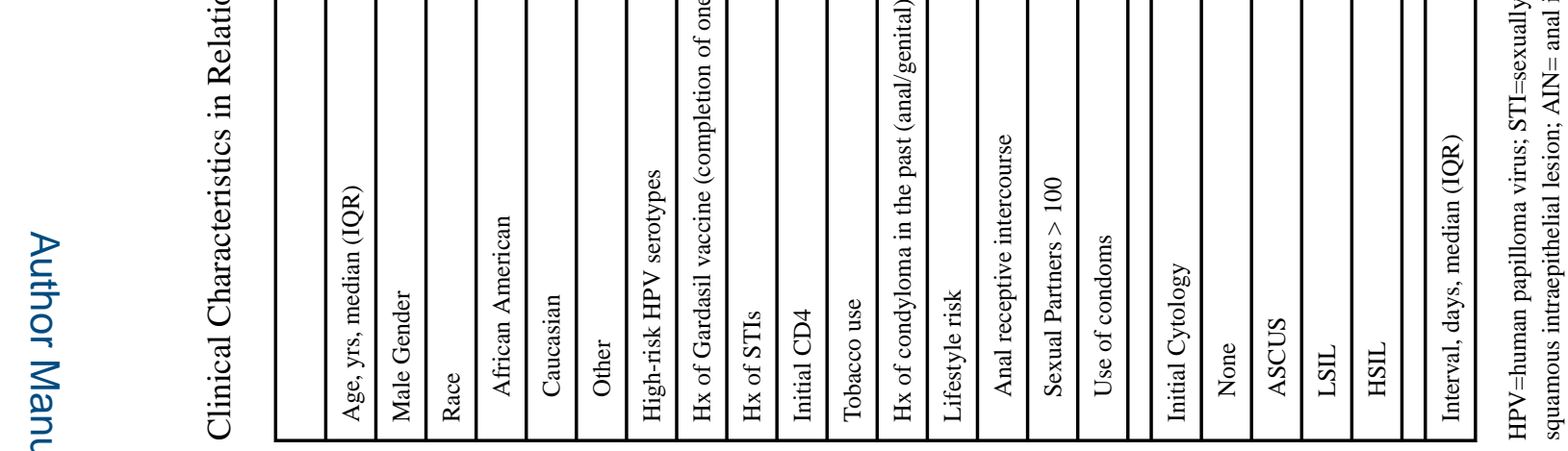

Tech Coloproctol. Author manuscript; available in PMC 2020 April 23. 